\title{
Évaluation des personnes en situation de handicap en milieu éducatif et professionnel : approche expérimentale
}

\author{
Eva Louvet et Odile roHMer \\ Université de Strasbourg \\ Faculté de psychologie
}

Résumé : En référence aux modèles actuels dans le domaine du jugement social, l'objectif de ce travail est d'analyser comment les personnes en situation de handicap sont jugées dans un contexte éducatif (Étude 1) et professionnel (Étude 2). Les participants sans handicap devaient évaluer des personnes avec ou sans handicap, sur des traits reflétant les principales dimensions du jugement social. Les résultats ont montré que les personnes avec handicap sont systématiquement jugées plus positivement sur la dimension de l'effort que sur la dimension de la compétence, alors que le pattern inverse a été observé pour les personnes sans handicap. Par ailleurs, la cible présentée avec handicap se voit attribuer un statut social moins élevé que la cible sans handicap. Ces résultats sont discutés au regard des actions à mettre en place pour favoriser l'inclusion sociale.

Mots-clés: Approche expérimentale - Contexte éducatif - Contexte professionnel - Évaluation - Inclusion sociale - Jugement social - Personnes en situation de handicap - Statut social.

Evaluation of persons with disability in an educational and in a professional context: an experimental approach

Summary: Building on recent research in the area of social judgment, this paper examines how persons with disability are evaluated in an educational (Study 1) and in a professional context (Study 2). Participants without disability had to rate persons presented with or without disability on traits reflecting the fundamental dimensions of social judgment. Our results showed that a person with disability was systematically judged more positively on effort than on competence, whereas the reversed pattern was observed for a person without disability. Furthermore, the target presented with disability was rated more negatively on social status. These results are discussed with regard to action to be undertaken to strengthen social inclusion.

Keywords: Educational context - Evaluation - Experimental approach - Persons with disability - Professional context - Social Inclusion - Social judgement - Social status.

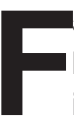

ace aux difficultés de participation sociale des personnes en situation de handicap, l'Organisation des Nations Unies a récemment rédigé une convention internationale relative aux droits de ces personnes. Cette convention ratifiée par plus de 150 pays dont la France, propose un cadre juridique dont l'objectif premier 
est de faire respecter le principe de non-discrimination. Dans cette perspective, les différents pays proposent des lois et actions positives (par exemple la loi 205-102 du 11 février 2005 sur les personnes handicapées en France), permettant à ces personnes d'accéder plus facilement à la formation et à l'emploi. Ces dispositifs ont effectivement permis aux jeunes en situation de handicap de bénéficier de soutiens humains et pédagogiques favorisant leur accès à l'enseignement (Ebersold, 2010 ; Le Roux et Marcellini, 2011). Néanmoins, en s'intéressant au devenir de ces jeunes, Ebersold (2010) note que l'accès facilité aux études ne se traduit pas de façon satisfaisante en termes d'accès à l'emploi. Dix ans après la promulgation de la loi de 2005, le taux d'emploi de cette catégorie de personnes stagne autour de $3 \%$ dans le secteur privé, donc bien en deçà de la volonté du législateur ${ }^{1}$. Ainsi, malgré une approche inclusive défendue par une société plus à l'écoute des besoins des personnes en situation de handicap, il semble donc persister un certain nombre de difficultés auxquelles sont confrontés les jeunes en situation de handicap et qui continuent à les exclure de la société.

Ces difficultés pourraient s'expliquer par des barrières psychosociales, liées aux représentations du handicap véhiculées dans notre société, difficiles à surmonter et résistantes aux politiques volontaristes de réduction des situations de handicap (Fougeyrollas, 1997 ; Molden et Tossebro, 2010 ; Watermeyer, 2012). Ce sont justement ces aspects plus subjectifs du processus de production et de maintien des situations de handicap que nos travaux tentent d'éclairer. Plus précisément, l'objectif de nos recherches est d'analyser le jugement social en tant que mécanisme psycho-social fondamental dans la détermination des conduites. En nous appuyant sur une démarche expérimentale, nous mesurons comment l'information être handicapé influence la façon dont on perçoit autrui dans deux contextes particulièrement déterminants pour l'inclusion sociale: l'enseignement et le travail. Notre objectif est de mettre en évidence que l'inclusion des jeunes en situation de handicap dans le système universitaire ne suffit pas pour transformer l'image de ces jeunes et les considérer de la même façon que les autres étudiants. Cette perception différenciée entre les jeunes en situation de handicap et les autres jeunes ne tend pas à diminuer au cours du temps et perdure dans le monde du travail.

\section{ComPétenCe et eFFort dans les modèles aCtuels de jugement soCial}

Pour étudier le processus de jugement, nous nous appuyons sur les modèles théoriques récents qui postulent que le jugement social repose sur deux dimensions fondamentales, liées d'une part aux capacités des personnes à établir et entretenir des relations sociales satisfaisantes (e.g. chaleureux, sympathique, honnête), d'autre part aux capacités des personnes à réussir sur le plan de leur vie professionnelle (e.g. intelligent, compétent, motivé) (Abele et Wojciszke, 2014; Dubois et Beauvois, 2005 ; Fiske, Cuddy, Glick, et Xu, 2002). Ces deux dimensions ont reçu différentes appellations. Dans le cadre de ce travail, nous les nommerons respectivement désirabilité sociale et utilité sociale (Dubois et Beauvois, 2005; Pansu et Dompnier, 
2011). De nombreux travaux expérimentaux mettent en évidence que les qualités reflétant l'utilité sociale différencient une personne qui réussit d'une personne qui échoue, alors que ces deux personnes sont jugées de manière équivalente sur les qualités associées à la désirabilité sociale (Dubois et Aubert, 2010 ; Louvet et Rohmer, 2010 ; Pansu et Dompnier, 2011). Lorsqu'on considère plus précisément les traits associés à l'utilité sociale, il semblerait que celle-ci recouvre deux facettes : d'une part la capacité à exhiber des compétences (efficace, intelligent, capable), et d'autre part la capacité à s'investir, à fournir des efforts (motivé, travailleur, persévérant). La complémentarité et la distinction de ces deux critères ont été largement développées pour modéliser un comportement efficace dans une organisation de travail (Curie, 1998 ; McCloy, Campbell, et Cudeck, 1994 ; Van Scotter et Motowido, 1996), ou pour expliquer la réussite et l'échec scolaire (Dompnier et Pansu, 2010 ; latridis et Fousiani, 2009; Weiner, 1986). En effet, les organisations de travail tout comme l'institution scolaire valorisent tout à la fois la compétence et l'effort comme deux qualités complémentaires nécessaires à la réussite.

Les travaux qui s'intéressent spécifiquement au jugement social à l'égard des personnes en situation de handicap montrent que ces personnes sont généralement perçues comme dépendantes, incompétentes, passives et faibles (Colella, De Nisi, et Varma, 1998; Gouvier, Sytsma-Jordan, et Mayville, 2003; Fichten et Amsel, 1986 ; Louvet, 2007 ; Louvet, Rohmer, et Dubois, 2009 ; Nario-Redmond, 2010 ; Ozawa et Yaeda, 2007). Cependant, d'autres travaux indiquent que le jugement à l'égard des personnes en situation de handicap n'est pas toujours négatif. Ces personnes se voient également attribuer certaines qualités telles que courageux, travailleur, persévérant et consciencieux (Christman et Slaten, 1991; Fichten et Amsel, 1986; Kurita et Kusumi, 2009; Louvet et Rohmer, 2010 ; Nario-Redmond, 2010 ; Rohmer et Louvet, 2011). En référence à la distinction entre compétence et effort et sur la base de ces résultats, on peut faire l'hypothèse que les personnes en situation de handicap sont dévalorisées sur la compétence, mais valorisées sur l'effort. L'objectif de notre recherche est de tester cette hypothèse dans un contexte éducatif et professionnel représentant deux contextes particulièrement déterminants pour refléter l'inclusion sociale dans notre société, autrement dit, deux contextes susceptibles de témoigner du succès des politiques d'inclusion. Si nous vérifions que les étudiants et les travailleurs en situation de handicap sont jugés moins compétents que leurs pairs sans que cette différenciation soit objectivement justifiable, il conviendra de s'interroger sur les politiques actuelles d'inclusion sociale.

\section{étude 1}

L'objectif de cette première étude est d'analyser la façon dont sont jugées des cibles fictives dans un contexte universitaire. Pour la moitié des participants, la cible est présentée comme un étudiant en situation de handicap (versus sans handicap). Le jugement porte sur les deux dimensions fondamentales relevées dans la littérature sur le jugement social, utilité sociale et désirabilité sociale (Dubois, 2006; Dubois et Beauvois, 2005). De façon novatrice par rapport aux travaux antérieurs, nous distinguons ici pour l'utilité sociale la compétence et l'effort. 
Méthode

Population 
Les participants étaient 57 étudiants sans handicap ( 26 hommes et 31 femmes) de l'université de Strasbourg, inscrits dans diverses filières (hors psychologie) et recrutés sur le campus universitaire pour prendre part à une recherche sur la formation des impressions. La participation à cette étude s'est faite sur la base du volontariat; elle était anonyme et non rétribuée. Les participants avaient entre 18 et 30 ans $(M=21.02 ; S D=2.32)$.

\section{Procédure}

Nous avons sollicité les étudiants pour participer à une enquête sur la façon dont on se forme une première impression sur autrui. Nous leur avons remis un livret qui contenait sur la première page les objectifs affichés de la recherche et nous permettait de recueillir des informations sur les participants. La deuxième page présentait une brève description d'un étudiant que les participants devaient lire attentivement, afin de se faire une première impression de la personne décrite. Enfin une dernière page contenait le questionnaire permettant de recueillir le jugement de l'étudiant décrit. La description de l'étudiant suivait le modèle suivant: "Philippe a 25 ans, il est célibataire et habite la région lyonnaise. Il poursuit actuellement des études dans le domaine de l'électronique analogique, une formation qui assure généralement de bonnes perspectives d'emploi. " Pour la moitié des participants, Philippe est présenté en situation de handicap: "À cause d'un accident de la circulation, Philippe est paraplégique et se déplace en fauteuil roulant. " La description se termine par: « Philippe a toujours réussi ses examens. Il pense vraiment avoir trouvé sa voie. " Après avoir lu attentivement le profil, les participants devaient indiquer les traits de personnalité qui, selon eux, décrivent le mieux Philippe. Pour répondre à cette question, ils disposaient de 15 traits et devaient indiquer « dans quelle mesure chacun d'eux caractérise Philippe », en se positionnant sur une échelle en six points allant de « pas du tout » à " tout à fait ». Parmi ces 15 traits, 5 renvoient à désirabilité sociale (agréable, aimable, chaleureux, gentil, sympathique), 5 à la compétence (compétent, compétitif, efficace, performant, productif) et 5 à l'effort (courageux, déterminé, méritant, motivé, persévérant). Afin de comparer le jugement des participants sur ces trois dimensions selon les conditions expérimentales, nous avons calculé trois scores composites correspondant à la moyenne des scores des cinq traits de chaque facteur (alpha Cronbach $=.86$ pour la désirabilité, .70 pour la compétence et .86 pour l'effort).

\section{Résultats et discussion}

Nous avons réalisé une analyse de variance introduisant la variable catégorie (avec ou sans handicap) comme facteur inter-sujets et les scores de jugement sur les trois dimensions considérées (désirabilité, compétence, effort) en mesures répétées. Cette analyse indique l'absence d'effet de la variable catégorie $(F(1,55)=2.51, p=.12)$ : L'étudiant en situation de handicap n'est pas globalement jugé plus négativement que l'étudiant sans handicap. Cependant, on peut observer une interaction entre 


\section{Méthode}

les variables catégorie et dimension du jugement $(F(2,110)=12.49, p<.0001$, Popylation 0.19 ). Les comparaisons de moyennes (cf. tableau 1) indiquent que l'étudiant en situation de handicap est jugé plus positivement sur la dimension de l'effort que sur la dimension de la compétence, et plus positivement sur la dimension de compétence que sur la désirabilité (LSD Fisher: $p<.01$ pour toutes les comparaisons). Par contre, l'étudiant présenté sans handicap est jugé plus positivement sur la dimension de la compétence que sur la dimension de l'effort, et plus positivement sur la dimension de l'effort que sur la dimension de la désirabilité ( $p<.01$ pour toutes les comparaisons). Autrement dit, dans les deux cas l'étudiant est mieux jugé sur les dimensions liées à l'utilité que sur la désirabilité, ce qui s'explique par le contexte de jugement. En effet, un étudiant qui semble réussir dans ses études se voit logiquement attribuer des qualités relevant de l'utilité sociale. Par contre, les qualités les plus mises en avant pour l'étudiant en situation de handicap ne sont pas les mêmes que celles mises en avant pour l'étudiant sans handicap : si un étudiant qui réussit est généralement surtout valorisé sur la compétence, un étudiant en situation de handicap qui réussit est d'abord valorisé sur l'effort. Tout se passe comme si pour réussir, un étudiant en situation de handicap devait déployer davantage d'efforts qu'un étudiant sans handicap. On pourrait imaginer que ce surcroît d'efforts vienne compenser un manque de compétence perçu. Dans la suite de ces résultats, la seconde étude est destinée à vérifier comment cette image de l'étudiant handicapé évolue quand il intègre le monde du travail.

Tableau 1 : Scores moyens de jugement (et écarts-types) des cibles présentées avec et sans handicap sur les trois dimensions de jugement (étude 1)

\begin{tabular}{|l|c|c|}
\hline & Sans handicap & Avec handicap \\
\hline Compétence & $4.99(0.90)$ & $4.85(0.66)$ \\
\hline Effort & $4.57(1.06)$ & $5.43(0.65)$ \\
\hline Désirabilité & $3.56(0.85)$ & $3.70(0.72)$ \\
\hline
\end{tabular}

\section{étude 2}

L'objectif de cette seconde étude est d'analyser la façon dont sont jugées des cibles fictives cette fois-ci dans un contexte professionnel. Pour la moitié des participants, la cible est présentée comme un travailleur en situation de handicap (versus sans handicap). Les mesures concernent à nouveau la compétence, l'effort et la désirabilité sociale attribués à la cible. Une mesure complémentaire porte sur le statut social attribué à la cible. Cette mesure nous permet de vérifier si la simple mention du handicap (toutes choses égales par ailleurs) induit la perception d'une position sociale inférieure. Cela nous permettra également de mettre en relation les deux facettes de l'utilité sociale (compétence versus effort) avec le statut perçu: quel est le rôle respectif de la compétence et de l'effort dans la prédiction de la réussite sociale? 
61 adultes tout venant et ne présentant pas de handicap, 36 femmes et 25 hommes âgés de 26 à 69 ans $(M=44.88 ; E T=10.45)$ ont été sollicités dans les lieux publics de Strasbourg pour participer à une étude sur la formation des impressions sur la base du volontariat. La participation à cette étude était anonyme et non rétribuée.

\section{Procédure}

Nous avons présenté aux participants une photo représentant une personne dans une situation de travail manuel ou intellectuel. Pour la moitié des participants, cette même personne était présentée dans un fauteuil roulant (cf. figure 1). La tâche des participants consistait à se forger une impression de cette personne afin de pouvoir porter un jugement sur elle. Pour exprimer leur jugement, les participants disposaient d'une liste de 9 traits de personnalité, et devaient indiquer dans quelle mesure chacune de ces traits caractérisait la personne photographiée, en se positionnant sur une échelle en neuf points allant de " pas du tout » à " tout à fait ». Parmi ces 9 traits, 3 renvoient à désirabilité sociale (aimable, chaleureux, sympathique), 3 à la compétence (compétent, efficace, productif) et 3 à l'effort (motivé, persévérant, volontaire). Afin de comparer le jugement des participants sur ces trois dimensions selon les conditions expérimentales, nous avons à nouveau calculé trois scores composites correspondant à la moyenne des scores des trois traits de chaque facteur (alpha Cronbach $=.88$ pour la désirabilité, .82 pour la compétence et .75 pour l'effort). Enfin, les participants devaient indiquer, toujours sur une échelle en neuf points, dans quelle mesure ils estimaient que la personne photographiée occupait un statut élevé (versus bas) au sein de l'organisation, et dans quelle mesure il avait beaucoup (versus peu) de responsabilités. Ces deux indicateurs étaient fortement corrélés $(r=.46 ; p<.0001)$, nous avons calculé un score moyen de statut perçu.

\section{Résultats et discussion}

Nous avons réalisé une première analyse de variance introduisant la variable catégorie (avec ou sans handicap) comme facteur inter-sujets et les scores de jugement sur les trois dimensions considérées (désirabilité, compétence, effort) en mesures répétées. Cette analyse indique l'absence d'effet de la variable catégorie $(F(1,59)$ $=3.15, p=.10)$ : la cible est jugée de la même façon, qu'elle soit présentée en fauteuil ou non. Cependant, comme dans la première étude, on peut observer une interaction entre les variables catégorie et dimension du jugement $(F(2,118)=9.21$, $p<.0002, \eta^{2}=0.13$ ). Les comparaisons de moyennes (cf. tableau 2 ) indiquent à nouveau que le travailleur présenté en fauteuil est jugé plus positivement sur la dimension de l'effort que sur la dimension de la compétence, et plus positivement sur la dimension de compétence que sur la désirabilité (LSD Fisher: $p<.01$ pour toutes les comparaisons). Par contre, si ce travailleur est présenté sans handicap, il est jugé plus positivement sur la dimension de la compétence que sur la dimension de l'effort, et plus positivement sur la dimension de l'effort que sur la dimension de la désirabilité $(p<.01$ pour toutes les comparaisons). Autrement dit, en milieu 


\section{Méthode}

professionnel comme en milieu scolaire, la cible est logiquement valorisée avant tout Populatiping dimensions liées à l'utilité sociale. Par contre, cette valorisation sur l'utilité sociale ne concerne pas les mêmes facettes selon que le travailleur présente ou non un handicap: s'il s'agit d'un travailleur en situation de handicap, la valorisation se fait surtout sur l'effort, alors que c'est la compétence qui est mise en avant, plus que l'effort, pour valoriser un travailleur (sans handicap).

Figure 1 : Matériel expérimental utilisé dans l'étude 2

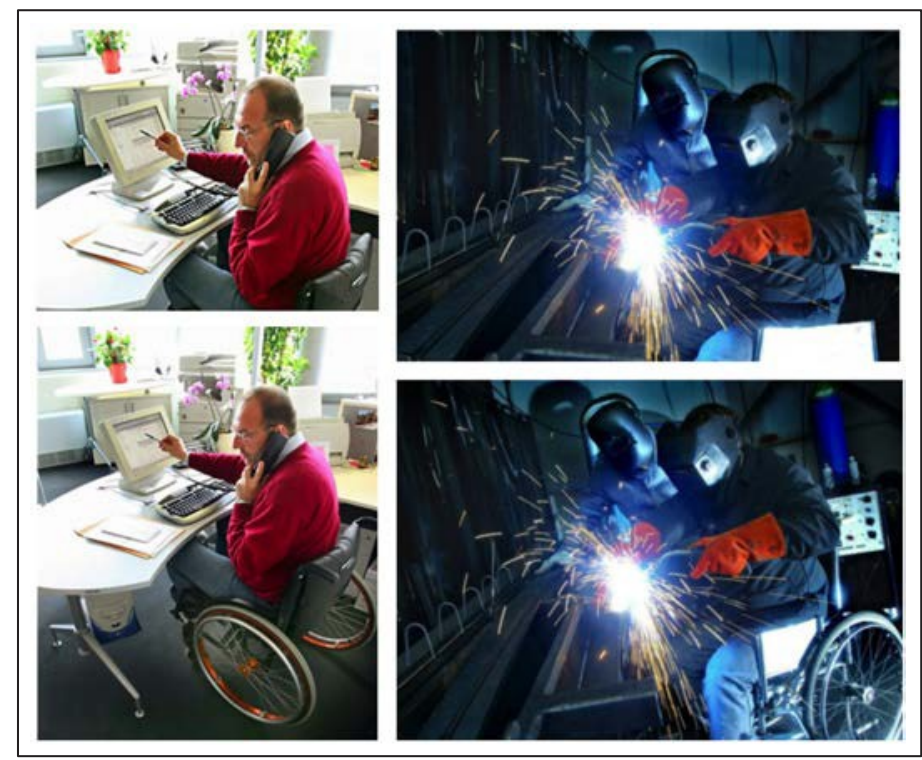

Tableau 2 : Scores moyens de jugement (et écarts-types) des cibles présentées avec et sans handicap sur les trois dimensions de jugement (étude 2)

\begin{tabular}{|l|c|c|}
\hline & Sans handicap & Avec handicap \\
\hline Compétence & $6.89(1.18)$ & $6.35(1.11)$ \\
\hline Effort & $6.19(1.52)$ & $7.20(0.84)$ \\
\hline Désirabilité & $4.42(1.54)$ & $5.16(1.28)$ \\
\hline Statut perçu & $4.87(1.61)$ & $4.03(1.47)$ \\
\hline
\end{tabular}

Une seconde analyse de variance nous permet de vérifier quelle est l'influence de l'information handicapé sur la perception du statut qu'une personne occupe dans le monde du travail. Les résultats indiquent que les participants attribuent en moyenne un statut plus élevé au travailleur présenté sans handicap comparativement au même travailleur présenté avec handicap $\left(F(1,59)=5.07, p<.03, \eta^{2}=0.07\right)$. Des analyses corrélationnelles mettant en relation les différentes dimensions du jugement avec le statut perçu révèlent que, conformément aux attentes, les deux facettes de l'utilité 
sociale sont positivement corrélées au statut perçu (pour la compétence $: r=.38$; $p<.003$; pour l'effort : $r=.26 ; p<.05)$ : plus on considère une personne compétente ou faisant des efforts, plus on l'imagine occuper un statut élevé dans son entreprise. Par contre, la désirabilité sociale est sans rapport avec le statut perçu. Enfin, une analyse de régression pas à pas ascendante permet d'évaluer l'impact respectif de la compétence et de l'effort pour prédire le statut. Cette analyse met en évidence que le statut perçu est prédit uniquement par la compétence, qui explique $14 \%$ de la variance totale $\left(F_{\text {incl }}(1,59)=9.81, p<.003 ; \beta=.37\right)$, alors que l'effort n'est plus lié au statut perçu $\left(F_{\text {incl }}(1,59)=0.82, p=.37\right)$. Autrement dit, les participants attribuent un statut d'autant plus élevé à une personne dans le monde du travail qu'ils jugent cette personne compétente. Par contre, le simple fait de l'imaginer faire des efforts n'implique pas l'attribution d'un statut élevé. Ce résultat est important lorsqu'on sait que les personnes en situation de handicap sont avant tout valorisées sur l'effort, alors que les personnes sans handicap le sont sur la compétence.

\section{disCussion générale}

L'objectif de ce travail était d'étudier l'image véhiculée par le handicap et de montrer en quoi cette image pourrait expliquer les difficultés d'inclusion persistantes auxquelles sont régulièrement confrontées les personnes en situation de handicap, alors que la France a mis en place une politique particulièrement volontariste pour encourager la participation sociale de ces personnes. Sur le plan méthodologique, nous avons adopté une démarche expérimentale qui présente l'avantage de pouvoir mesurer en quoi la simple mention du handicap, toutes choses égales par ailleurs, transforme la façon dont on perçoit un individu. En contexte plus écologique, il est généralement difficile de distinguer ce qui relève de l'intention de juger négativement des personnes sur la simple base du handicap, de ce qui relève de caractéristiques différenciant objectivement les personnes en situation de handicap des personnes sans handicap. En effet, statistiquement, les personnes en situation de handicap disposent en moyenne d'un niveau de formation moins élevé, ont moins d'expérience professionnelle, sont plus âgées, moins mobiles et moins autonomes que le reste de la population (Ebersold, 2010 ; Lo et Ville, 2013). Par conséquent, il est difficile de savoir dans quelle mesure ces caractéristiques expliquent la mise à l'écart de ces personnes, indépendamment d'une discrimination intentionnelle (Ravaud, 1998). Seule la méthode expérimentale permet d'isoler le facteur handicap des autres caractéristiques qui pourraient lui être associées.

Dans nos recherches, nous avons présenté aux participants la description d'une cible fictive à partir d'un petit texte ou d'une photo, en modifiant uniquement l'information relative au handicap (présente ou absente). Les participants devaient évaluer cette cible à partir d'une série de traits de personnalité recouvrant les deux dimensions fondamentales du jugement: l'utilité sociale (décomposée en deux sous-dimensions : la compétence et l'effort) et la désirabilité sociale (Dubois et Beauvois, 2005; Louvet et al., 2009). Ces études ont été contextualisées dans deux secteurs particulièrement importants pour favoriser l'inclusion sociale: le contexte éducatif et le contexte professionnel. Les résultats de ces deux études sont convergents et confirment que la mention du handicap induit en soi une perception différente de la personne. Lorsque 
la personne est présentée en situation de handicap (moteur), elle est surtout perçue comme une personne faisant des efforts, alors que lorsque cette même personne est présentée sans handicap, elle est surtout perçue comme compétente. Par ailleurs, nos résultats ont clairement confirmé que seule l'évaluation des compétences prédit le statut qu'on imagine occupé par une personne: plus on imagine une personne compétente, plus on l'imagine haut placée dans la hiérarchie sociale. Par conséquent, du fait qu'on valorise les personnes en situation de handicap avant tout sur l'effort et non pas sur la compétence, on leur attribue un statut moins élevé. L'ensemble de ces résultats illustrent ainsi la façon dont nos croyances subjectives, c'est-à-dire non fondées sur des informations objectives, peuvent défavoriser les personnes en situation de handicap et limiter leurs chances de participer pleinement à la vie de notre société. Malheureusement, ces barrières psycho-sociales auxquelles sont confrontées les personnes handicapées sont insidieuses dans la mesure où elles sont à la fois difficiles à mettre en évidence sur le terrain et difficiles à combattre.

\section{ConClusion}

Les deux études présentées ici témoignent de la prégnance des barrières psychosociales et des dangers qu'elles représentent pour que les politiques d'inclusion puissent être vraiment efficaces. Allant dans ce sens, la nécessité de prendre en considération les processus psychologiques, pour œuvrer vers une meilleure participation sociale des personnes en situation de handicap, a récemment été soulignée dans la littérature : Watermeyer (2012) met en avant que le modèle social du handicap, tel qu'il est promu par l'Organisation mondiale de la santé, néglige la prise en compte des préjugés et croyances qui entravent le processus de participation sociale. La lutte contre ces phénomènes n'est certes pas évidente, elle passe néanmoins par une meilleure information, une diffusion des résultats de recherche et sans doute aucun, par une mixité des publics scolarisés ensemble dès l'enfance. Cette mixité doit impliquer des situations d'apprentissage ou de travail où les jeunes en situation de handicap puissent valoriser leurs compétences. 


\section{Références bibliographiques}

Abele, A., \& Wojciszke, B. (2014). The big two in social judgment and behavior. Social Psychology, 44, 61-62.

Christman, L. A., \& Slaten, B. L. (1991). Attitudes toward people with disabilities and judgments of employment potentials. Perceptual and Motor Skills, 72, 467-475.

Colella, A., De Nisi, A.S., \& Varma, A. (1998). The impact of ratee's disability on performance judgments and choice as partner. Journal of Applied Psychology, 83, 102-111.

Curie, J. (1998). Le discours de la compétence ou l'expert et la diseuse de bonne aventure. Éducation permanente, 135, 133-142.

Dompnier, B., et Pansu, P. (2010). La valeur sociale des explications causales en contexte éducatif : Autoprésentation des élèves et représentation des enseignants. Swiss Journal of Psychology, 69, 39-51.

Dubois, N. (2006). La valeur sociale des personnes. In R.V. Joule et P. Huguet (eds), Social Psychology (pp. 81-116). Grenoble: PUG.

Dubois, N., et Aubert, E. (2010). Valeur sociale des personnes: deux informations valent-elles mieux qu'une? Revue internationale de psychologie sociale, 23, 57-92.

Dubois, N., \& Beauvois, J. L. (2005). Normativeness and individualism. European Journal of Social Psychology, 35, 123-146.

Ebersold, S. (2010). L'accès à l'enseignement supérieur des étudiants handicapés dans certains pays de l'OCDE: enjeux et perspectives. La nouvelle revue de l'adaptation et de la scolarisation, 50, 165-174.

Fichten, C.S., \& Amsel, R. (1986). Trait attributions about college strudents with a physical disability: circumplex analyses and methodological issues 1. Journal of Applied Social Psychology, 16, 410-427.

Fiske, S.T., Cuddy, A.J., Glick, P. \& Xu, J. (2002). A model of (often mixed) stereotype content: Competence and warmth respectively follow from perceived status and competition. Journal of Personality and Social Psychology, 82, 878-902.

Fougeyrollas, P. (1997). Les déterminants environnementaux de la participation sociale des personnes ayant des incapacités. Canadian Journal of Rehabilitation, 10, 147-160.

Gouvier, W.D., Sytsma-Jordan, S., \& Mayville, S. (2003). Patterns of discrimination in hiring job applicants with disabilities: the role of disability type, job complexity, and public contact. Rehabilitation Psychology, 48, 175-181.

latridis, T., \& Fousiani, K. (2009). Effects of status and outcome on attributions and just-world beliefs: How the social distribution of success and failure may be rationalized. Journal of Experimental Social Psychology, 45, 415-420.

Kurita, T., \& Kusumi, T. (2009). Implicit and explicit attitudes toward people with disabilities and effects of the internal and external sources of motivation to moderating prejudice. Psychologia, 52, 253-260. doi: 10.2117/psysoc.2009.253

Le Roux, N., et Marcellini, A. (2011). L'insertion professionnelle des étudiants handicapés en France. Revue de questions et axes de recherche. European Journal of Disability Research, 5, 281-296. 
Lo, S.H., \& Ville, I. (2013). The "employability" of disabled people in France: A labile and speculative notion to be tested against the empirical data from the 2008 "Handicap-Santé" Study. European Journal of Disability Research, 7, 227-306.

Louvet, E. (2007). Social judgment toward job applicants with disabilities: Perception of personal qualities and competences. Rehabilitation Psychology, 52, 297-303.

Louvet, E., et Rohmer, O. (2010). Les travailleurs handicaps sont-ils perçus comme des travailleurs compétents ? Psychologie du Travail et des Organisations, 16, 47-62.

Louvet, E., Rohmer, O., \& Dubois, N. (2009). Social judgment of people with a disability in the workplace: how to make a good impression on employers? Swiss Journal of Psychology, 68, 153-159.

McCloy, R.A., Campbell, J.P., \& Cudeck, R. (1994). A confirmatory test of a model of performance determinants. Journal of Applied Psychology, 79, 493-505.

Molden, T.H., \& Tossebro, J. (2010). Measuring disability in survey research: Comparing current measurements within one data set. European Journal of Disability Research, 4, 174-189.

Nario-Redmond, M.R. (2010). Cultural stereotypes of disabled and non-disabled men and women: consensus for global category representations and diagnostic domains. British Journal of Social Psychology, 49, 471-488.

Ozawa, A., \& Yaeda, J. (2007). Employer attitudes toward employing persons with psychiatric disability in Japan. Journal of Vocational Rehabilitation, 26, 105-113.

Pansu, P., \& Dompnier, B. (2011). A bi-dimensional scale of scholastic value: social desirability and social utility, two dimensions of personological judgment. European Review of Applied Psychology, 61, 31-41.

Ravaud, J.F. (1998). Handicap et emploi: la question des désavantages. In A. Blanc et H.J. Stiker, Linsertion professionnelle des personnes handicapées en France (pp. 107-129). Paris: Desclée de Brouwer.

Rohmer, O., et Louvet, E. (2011). Le stéréotype des personnes handicapées en fonction de la nature de la déficience. Une application des modèles de la bi-dimensionnalité du jugement social. L'année psychologique, 111

Van Scotter, J.R., \& Motowido, S.J. (1996). Interpersonal facilitation and job dedication as separate facets of contextual performance. Journal of Applied Psychology, 81, 525-531.

Watermeyer, B. (2012). Is it possible to create a politically engaged, contextual psychology of disability? Disability and Society, 27, 161-174.

Weiner, B. (1986). An attributional theory of motivation and emotion. New-York: Springer. 